To the Editors:

\title{
A case of Kleine-Levin syndrome
}

Ceylon Medical Journal 2011; 56: 132-133

Kleine-Levin syndrome is a rare sleep disorder predominantly described in males [1]. It has a relatively good prognosis and awareness would avoid inappropriate management as either a psychotic disorder or epilepsy.

A 12-year old prepubertal girl presented with episodes of hypersomnolence, associated with megaphagia and altered behaviour of 4 years duration. A typical episode starts with headache and facial pain, followed by increased sleepiness lasting 3-4 days, associated with hyperphagia and emotional lability.
Examination showed a well child who preferred to sleep all the time (18-22 hours/day). She had stable vital signs, without focal neurological signs and normal systemic examination.

Electroencephalogram performed during an episode showed no seizure activity. Treatment with sodium valproate also showed no response. Inborn errors of metabolism were excluded by normal urine screen for aminoacidopathies and organic acidaemias and normal serum ammonia, lactate, and blood gases during two clinical episodes. Her blood glucose, liver and renal 
functions, inflammatory markers (CRP, ESR), cerebrospinal fluid analysis and MRI scan of brain were normal.

The presence of unusual triad of hypersomnolence, hyperphagia associated with abnormal behaviour as the predominant symptoms, in the presence of negative investigations lead to the clinical diagnosis of Kleine-Levin syndrome in this child.

Kleine-Levin syndrome (KLS) is a rare but relatively benign syndrome characterised by recurrent episodes of hypersomnia and at least one of the following symptoms: (1) cognitive or mood disturbances, (2) megaphagia with compulsive eating; (3) hypersexuality with inappropriate behaviours; and (4) abnormal behaviour [1]. These episodes are separated by weeks or months of normal sleep and behaviour. Though originally described only in adolescent males, rarely young females are affected. A complete syndrome is the occurrence of hypersomnia, megaphagia and the various psychic manifestations. However atypical and incomplete forms are described. The episodes occur suddenly and last for several days to weeks, and cease abruptly. The interparoxysmal periods last several days to months, sometimes even to several years. Duration of each symptomatic period reduce over the years. The exact pathogenesis is not completely understood. A disorder of the diencephalon with episodic diffuse brain hypoperfusion, a viral aetiology due to associated flu like symptoms or autoimmune basis due to association with certain HLA types are postulated [2,3].

Many therapeutic agents such as stimulants, mood stabilisers, amphetamines and neuroleptics have been used, but definite benefit is yet to be established. With increasing age, frequency of these episodes gradually decrease. Therefore management during each episode is primarily supportive and educational [4].

\section{References}

1. Critchley M. Periodic hypersomnia and megaphagia in adolescent males. Brain 1962; 85: 627-56.

2. Huang YS, Guilleminault C, Kao PF, Liu FY. SPECT findings in the Kleine-Levin syndrome. Sleep 2005; 28: 955-60.

3. Dauvilliers Y, Mayer G, Lecendreux M, et al. Kleine-Levin syndrome: an autoimmune hypothesis based on clinical and genetic analyses. Neurology 2002; 59: 1739-45.

4. Arnulf I, Lin L, Gadoth N, et al. Kleine-Levin syndrome: a systematic study of 108 patients. Annals of Neurology 2008; 63: 482-93.

\author{
J Wanigasinghe ${ }^{1}$, S G Mettananda ${ }^{2}$, D S Kodikara Arachchi ${ }^{3}$ \\ ${ }^{1}$ Department of Paediatrics, Faculty of Medicine, University of Colombo, ${ }^{2}$ Department of Paediatrics, Faculty of \\ Medicine, Ragama, ${ }^{3}$ Teaching Hospital, Karapitiya, Galle, Sri Lanka.
}

Correspondence: JW, e-mail: <jithwani@yahoo.com>. Received 18 August 2010 and revised version accepted 1 March 2011. Competing interests: none declared. 